\title{
IV. Weitere Beiträge zur Aetiologie der Infectionskrankheiten.
}

VII.

Zur Aeliologie der Lungenentzündung.

1) J. Kerschensteıner, leber infectiöse Pneumonıe, Vortrag, geh. im ärztl. Verein zu München am 1. Dec. 1880. Sep.-Abdr. -

2) Köhnhorn, Zur Aetrologie der Lungenentzündung. VJS. f. ger. Med. und öff. San.-Wesen 1881, Juli.

3) Butry, Leber eine maligne Pneumonie-Epideme Im Dorfe Becherbach. Deutsches Arch. f. klin. Medicin. Bd. XXIX, H. 1 u. 2.

Referent A. Wernich.

1) In der Männergefangenenanstalt zu Amberg erkrankten vom 1. Jan. bis zum 28. Mai 1881 bei einer Gesammtzahl von 1200 Sträflıngen $161 \mathrm{~d}$. h. ungefähr jeder sıebente Sträflıng an Pneumonie; $46=28, \tilde{3}$ Proc. der Erkrankten starben. Bei dem unzweifelhaften Sectionsbefunde der croupösen Lobärpneumonie mit rother oder grauer Hepatisation und theilweiser Eiterbildung waren die klinischen Erscheinungen ebenfalls nur insofern abweichende, als eine gewisse Asthenicität in der Entwicklung und besonders in der Genesungsperıde sıch geltend machte. Weder von einem Erkàltungseinfluss noch von einer nnmittelbaren ursächlichen Einwirkung der Beschäftigungsweise bonnte die Rede sein; ebenso ergab die Beschaffenheit der Kost und des Trinkwassers nicht den geringsten Anhalt für die Krankheitsentstehung. Fom Aufsichtsund Wartepersonal erkrankte Niemand. Die Aufmerksambeit lenkte sich hiernach speciell auf die Schlafsäle der Gefangenen; keine dieser Localitäten, sowelt sie in Benutzung waren, zeıgte sich voll Erkrankungen frei; die Dichtıgkeit der Belegrung und die Zahl der Betten stand nicht in directenı Verhältniss zur Zalıl der Erkrankungsfälle; die Anordnung der Ahorte sclien ebenfalls nıcht direct verantwortlich, da gerade dem am messten betroffenen Schlafsaal der Abort nicht angrenzte. Klar erwies sich nur, dass die anı bürzesten in Haft befindlichen Gefangenen an meisten zur Lungenentzündung geneigt waren und bei einer historschen Nachforschung, dass die, Lungen- und Brustbrankheiten in der Anstalt schon immer epidemisch und schubweise aufgetreten waren. K. hommt zu dem Schluss: „Es blebbt nur übrig ein nicht transportabler Krankheitserreger, der an der Localität haftet, ene Art Malaria, jedenfalls etwas, was man jetzt noch bes dem dermalıgen Sprachgebrauche mit dem Nanen ..Mlasma“" zu bezeichnen berechtigt ist."

2) Köhnhorn seinerselts gewann aus der Art des Auftretens der croupösen Pneumonie unter den Truppentheilen und in den Casernen zu Wesel Gründe für die Anschauung, dass diese Art der Lungenentzündungen nicht als „Erkàltungsleiden", sondern als Infectıonskranhlieıt aufgefasst werden müsse. Seine Tabellen zeigen, dass nicht die kalten Monate dıe grössere Zahl der Pneunıniefälle aufweısen; auch dass nıchı plötzlıche Temperatursprünge, nicht die Windrıchtung, nıcht der 0zongehalt der Luft, noch das Scliwanken des Grundwassers die constaturte Verschiedenheit der Pneumoniefrefuenz in den einzeluen ilonaten erklären hönnen. Vielmehr wurden Truppentheile, welche längrese Zett freigeblieben waren, plötzlich in grosser Zahıl won Pnemnone heimgesucht, und andere Abtheilungen blieben gleichzeitigr - sownhl 1 ul sommer wie im Winter - gänzlich verschıout. An die Anstren:"ungen in Dienst war das Erscheinen der Krankheıt elenfalls niclt getinüpt, eher an gewisse hasernen, in denen sie endemisch eıngenıstet schien und sich jeweilig zu wohlausgesprochenen lacalen Epidenien

1) Demgemäss dürfte die Pinselung beı allen jenen Zustälıden contraindicirt sein, bei welchen eine Erkrankung der NN. optici unter dem Bild einer langsam fortschreitenden weissell Atrophie ohne beträchtlıche Gefassinjection einhergeht, ein schluss, welcher sich auch durch die Erfahrung bei Pinselung in Folge gleichzeitiger anderer Affection be. stätigte. 
steigerte. - Auflällig war der Thatbestand, der sich herausstellte, als K. mit der Verthellung der Pneumonien auf die einzelnen Kasernen die Häufigkeit der Wechselfieber in denselven verglich: Innerhalb eines Zeitraums von 8 Jahren entfielen 308 Fälle von Pneumonien und 305 von Wechselfiebern zu ganz überraschend gleichen Procenten a uf dieselben Kasernenlocale. - Dagegen gewährten die Zahlen Kölınhorn's keinen Anhalt für dıe Behauptung Jürgensen's, dass die Jahre mit einer grossen Frequenz an Typhen auch die grösste Häufigkeit der Lungenentzündungen zeigen sollen.

3) Mit eıner Heftigkett nnd Bösartigkett, wie noch nie in der ganzen Gegend, trat in Dorfe Becherbacli des Kreises Meisenhein im März und April dieses Jahres eine Enidemie von Lungenentzündungen auf, welclie von 460 Bewohnern $20=4$ Proc. ergriff. Von den nnter 15 Jahren alten 8 Erkrankten starb nur 1; von den älteren 12 dagegen 8 , also beinahe 70 Proc. der letzten Categorıe. Die Krankheit suchte besonders enzelne Familien heim, welche unter sich nachbarhche Beziehungen nnterhelten, nod hatte in den meisten Fällen deu ,atypischen scliweren Charakter sogenannter astlienischer oder typhöses Pneumonien". Sectionen sind leıder nicht zu ermöglichen gewesen. 